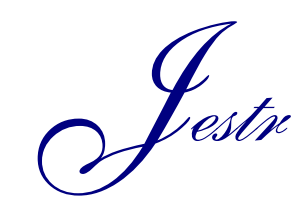

Journal of Engineering Science and Technology Review 12 (2) (2019) 111 - 118

JOURNAL OF

Engineering Science and

Technology Review

Research Article

www.jestr.org

\title{
Internal Model Control of Discrete Non-Minimum Phase Over-Actuated Systems with Multiple Time Delays and Uncertain Parameters
}

\author{
Islem Bejaoui $^{1, *}$, Imen Saidi ${ }^{1}$, Maria Gabriella Xibilia ${ }^{2}$ and Dhaou Soudani ${ }^{1}$ \\ ${ }^{1}$ U.R. LARA Automatique, Ecole Nationale d'Ingénieurs de Tunis, University of Tunis El Manar, BP 37, Le Belvédére,1002 Tunis, \\ Tunisia \\ ${ }^{2}$ Dipartimento di Ingegneria, Università degli studi di Messina, ITALY
}

Received 7 January 2019; Accepted 10 April 2019

\begin{abstract}
: Internal model control (IMC) is an established technique in continuous time linear control, but it is less used for discrete-time systems. Most of the existing solutions do not cover all the situations and, in any case, they lead to complex procedures to design the controller. In this paper, a IMC technique able to control over-actuated systems is used to deal with a discrete-time Non-Minimum-Phase (NMP) process with multiple time delays and uncertain parameters. The proposed IMC control scheme is based on the system augmentation with a suitable number of virtual outputs to the model matrix, in order to create a square matrix, so that the realization of an approximate inverse of the model plant is possible. Robust stability analysis is provided via combination of the value set concept and the zero exclusion conditions. Internal stability is verified using Linear Matrix Inequalities (LMI). Simulations are reported to demonstrate the suitability of the proposed design, as regards robust stability, performance, parametric uncertainties and load disturbances.
\end{abstract}

Keywords: Internal model control; LMI stability; parametric uncertainty; Value set concept; Zero Exclusion Condition; Over-Actuated Systems.

\section{Introduction}

The processes with more inputs than outputs, known as over-actuated systems, are difficult to control due to the presence of the interactions between several outputs variables and the manipulating inputs channels. This class of system arises in a wide range of applications including many chemical processes, (chemical reactors, distillation column), thermal systems, (heat exchangers), road vehicles, mobile robots, transportation and communication systems, etc ... [1].

The large majority of processes are multivariable in nature, exhibiting some strong coupling and occasionally a non-minimum phase (NMP) character that makes the control design problem a challenging task [2]. Discrete system having at least one transmission zero outside the unit circle are termed as NMP systems [3]. For instance, the invertible minimum phase (MP) system can provide good regulation, which an NMP system lacks, these systems can become unstable in closed-loop form. This makes some NMP systems almost uncontrollable [4]. Despite the coupling problems to maintain several controlled variables at independent set points with over-actuated systems, a nonminimum phase system with time delays and parameter uncertainty is even more difficult to control.

When employ controllers which require the inverse model of the system, is possible to talk about the internal model control (IMC), which was introduced in the 80 's by Manfred Morari and his co-workers, (Morari and Zafirou

*E-mail address: islem.bejaoui@enit.utm.tn

ISSN: $1791-2377$ @ 2019 Eastern Macedonia and Thrace Institute of Technology. All rights reserved. doi:10.25103/iestr.122.16
1989, Garcia and Morari 1982 etc), has already proved to provide an effective framework for robust control of various classes of delay-free systems [5]. It is therefore usual to extend this concept to control over-actuated systems.

IMC has been proved to be successful in a wide area of control applications, such as process control [6], electrical drive systems [7], signal processing and power electronics [8] and [9]. Internal model control (IMC) structure is derived from classical control by introducing the model of process in the control loop and thereby having significant advantages over classical control such as stability, perfect control loop and zero-steady state offset. The basic one degree of freedom IMC provides good compromise between set-point tracking and disturbance rejection and works well for NMP system [10]. Pedro [11] proposed an output estimator for NMP MIMO plants. The non-delayed output plant is predicted. The predictor is a stable dead-time-compensator coping with multiple and arbitrary delays in all the signal channels. The controller is designed for the resulting nondelayed plant and the proposed scheme is based on a new smith predictor.

Jing [12] proposes a simple design method of IMC for NMP non-square processes with different time delays, it is realizable by adding additional low-pass filters and provides a model reduction method to find a good approximation of the theoretical controller.

Jian [13] presented a modified two-degrees-of-freedom IMC for non-square systems with multiple time delays and right-half-plane zeros. In this approach, pseudo-inverse method is used to design the internal controller. The obtained control system was sensitive to the change of time delays. 
Islem Bejaoui, Imen Saidi, Maria Gabriella Xibilia and Dhaou Soudani/

Journal of Engineering Science and Technology Review 12 (2) (2019) 111 - 118

Ricardo [14] present an internal model control based proportional-integral-derivative (PID) control for a benchmark system presented at the $3^{\text {rd }}$ IFAC conference on advances in proportional-integral-derivative control (PID18). The design of the internal controller was based on a linearized model of the benchmark system around the normal operation point, using the prediction error estimation algorithm, then the interaction effects are neglected based on decentralized approach. The authors in this paper are carried out to solve the application problem of PID controller in MIMO systems, the IMC based PID controller validate the performance of the proposed method.

In this paper, the approach previously developed in [15] and [16] is extended to NMP discrete-time over-actuated systems with time delays. Robust stability is also analyzed by considering parameters uncertainty.

IMC based virtual outputs controller for over-actuated systems is proposed. Firstly, using a suitable number of virtual outputs, an augmented model is obtained to ensure the approximate model inversion and to deal with the overactuation problem. Then the virtual adding will be neglected. The stability of the proposed controller is analyzed using the matrix inequality approach (LMI) [17] and the internal stability of IMC structure in the presence of uncertainty at the level of the system is verified using the graphical approach [18]. IMC controller based on virtual outputs is designed for non-square system to validate the results of the proposed approach

This article is structured as follows. In section 2 the approach for an internal model controller design, directly applicable to discrete-time over-actuated systems, is described, and the augmented model is designed. The robust stability approach for system with uncertainty parameters used in the paper is reported in section 3 . In section 4 , an example is reported to illustrate the efficiency of the proposed controller. Some conclusions are given in section 5.

\section{Internal model control design for over-actuated systems}

\subsection{The Virtual Outputs approach}

The basic control block diagram of IMC structure for multivariable systems is shown in Figure 1, where G(z) and $\mathrm{M}(\mathrm{z})$ are the process and the process model respectively. $\mathrm{C}(\mathrm{z})$ is the internal model controller [19].

If the process model is perfect, the difference between process output and model output (i.e., feedback signal) is not affected by the action of the manipulated variables but it is influenced only by the disturbance. Consequently, the control system is effectively open-loop and C(z) plays the role of feedforward controller.

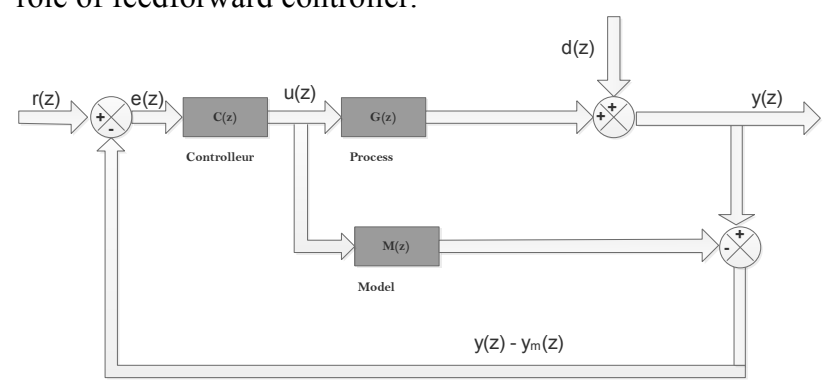

Fig. 1. The IMC basic structure

The synthesis of an IMC controller $\mathrm{C}(\mathrm{z})$ is equal to the inverse of the internal model $\mathrm{M}(\mathrm{z})$ with more control inputs (m) than outputs (n) in order to ensure a perfect set-point tracking. The approximate inverse represents the major problem of the IMC approach, because the realization of the direct inversion is difficult for non-square systems (rectangular matrix) [19]. This difficulty becomes more complicated in the presence of unstable zeros or/and time delay.

A new inversion technique of the over-actuated model $\mathrm{M}(\mathrm{z})$ using virtual outputs is proposed. It consists on attaching lines to the rectangular transfer function matrix of the process model, which allows the inversion technique to be realizable. To maintain the original characteristics of the process, we have to remove the adding lines. The virtual outputs are only used to square the model transfer matrix [20].

Once the virtual outputs technique is applied, we need now to modify the basic IMC structure previously mentioned in Figure 1, so that it becomes applicable to overactuated systems.

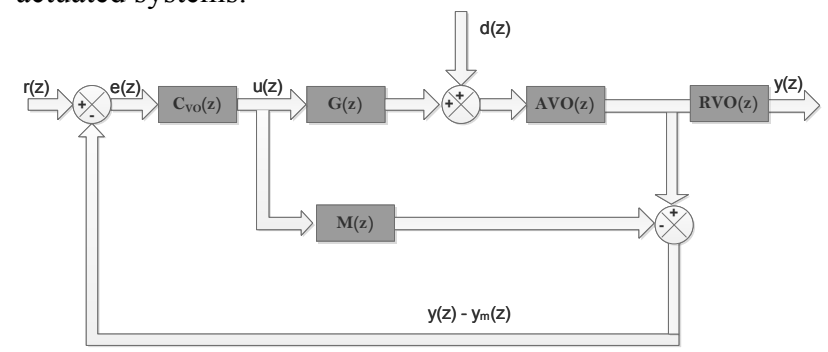

Fig 2. The proposed IMC design for discrete over-actuated systems

The modified IMC structure presented in Figure 2 is characterized by two more blocks [20], [21]. The first added one present the Augmentation Virtual Outputs AVO(z) block which is used to augment the model outputs inserting virtual $((m-n) m)$ lines to the transfer matrix of the rectangular process model in order to make it of dimension $(m \times n)$, so that it can be inverted. The second added one is the Removing Virtual Outputs RVO (z) block which is used to eliminate the exceeding virtual outputs by means of the usual logical operators [21].

$\mathrm{G}(\mathrm{z})$ is a process with ' $\mathrm{m}$ ' inputs and ' $\mathrm{n}$ ' outputs $(m\rangle n)$ given as:

$G(\mathrm{z})=\left(\begin{array}{cccc}g_{11}(\mathrm{z}) & g_{12}(\mathrm{z}) & \ldots & g_{1 m}(\mathrm{z}) \\ g_{21}(\mathrm{z}) & g_{22}(\mathrm{z}) & \ldots & g_{2 m}(\mathrm{z}) \\ \vdots & \vdots & \ddots & \vdots \\ g_{n 1}(\mathrm{z}) & g_{n 2}(\mathrm{z}) & \ldots & g_{n m}(\mathrm{z})\end{array}\right)$

The virtuals $((m-n), m)$ added lines are expressed as:

$$
G_{+}(\mathrm{z})=\left[\begin{array}{cccc}
g_{n+1,1}(z) & g_{n+1,2}(z) & \ldots & g_{n+1, \mathrm{~m}}(z) \\
g_{n+2,1}(z) & g_{n+2,2}(z) & \ldots & g_{n+2, \mathrm{~m}}(z) \\
\vdots & \vdots & \ddots & \vdots \\
g_{m 1}(z) & g_{m 2}(z) & \ldots & g_{m m}(z)
\end{array}\right]
$$

where the transfer function from $\mathrm{j}$-th input to $\mathrm{i}$-th output is $g_{i j}(z)=g_{0 i j}(z) z^{-\tau i j}, \quad g_{0 i j}$ is strictly proper, stable and 
possible non-minimum phase and $\mathrm{t}_{i j}$ is the time delay which is a non-negative constant. $g_{0 i j}(z)$ is defined as

$g_{i j}(z)=\frac{K_{i j} N_{N M P}(z) N_{M P}(z)}{D_{s}(z) D_{u}(z)}$

where, $K_{i j}$ is the gain of the each $g_{i j}(\mathrm{z}), N_{N M P}(\mathrm{z})$ and $N_{M P}(z)$ are the non-minimum phase $(N M P)$ and minimum phase $(M P)$ numerator polynomials. $D_{s}(z)$ and $D_{u}(z)$ are the stable and unstable denominator polynomials. The presence of non-minimum phase elements, namely time delays limits the ability for perfect control.

However, the control object in our case is an overactuated system that can be $N M P$, so it does not have the traditional inverse. Thus, the Virtual Outputs approach is introduced. The virtual output controller $C_{v o}(z)$ presented in Figure 3 is expressed as:

$$
C_{V O}(z)=u e^{-1}=\left(\mathrm{I}_{\mathrm{m}}+K_{1} M(\mathrm{z})\right)^{-1} K_{1}=\left(K_{1}^{-1}+M(\mathrm{z})\right)^{-1}
$$

where $I_{m}$ is the identity matrix and $K_{1}$ is an invertible square diagonal matrix; it must ensure the stability conditions of the controller [22]. $K_{1}$ is an invertible ( $m \times m$ ) square matrix, it can be expressed by:

$K_{1}=\beta \times \mathrm{I}_{\mathrm{m}}, \beta \in R^{+}$
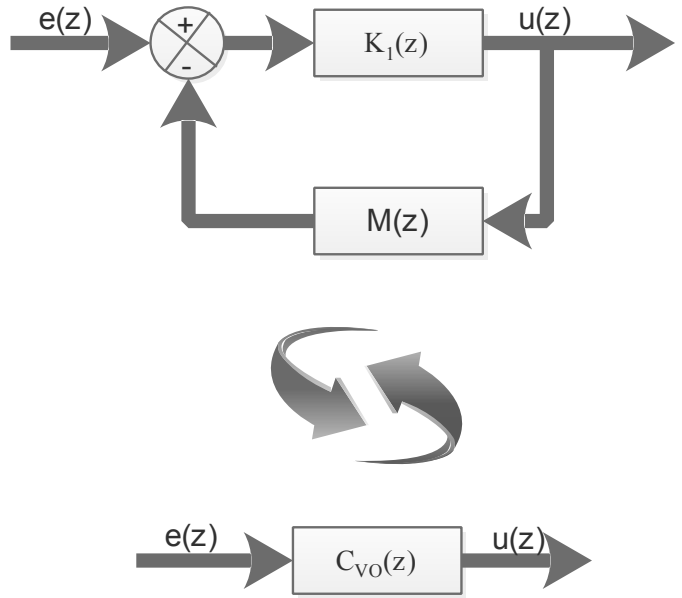

Fig. 3. Virtual Output Controller structure

If we choose a high value of $b$ in (5), we obtain a small value of $\frac{1}{\mathrm{~b}}$ which allows to approximate $\left(K_{1}^{-1}+M(z)\right)^{-1}$ with $M(z)^{-1}$. In this case, $C_{v o}(z)$ is approximately equal to the model expression inverse as follows:

$C_{V O}(z)=M(\mathrm{z})^{-1}$

Proof (case of a square system $(2 \times 2)$ )

We consider a multivariable discrete system with 2-inputs, 2-outputs, whose the matrix $M(z)=\left(m_{i j}(z)\right)_{i, j=1,2}$ is the transfer matrix relating to the model describing the system, and $K_{1}=\beta I_{2}$.

The transfer matrix of the controller $C_{v o}(z)$ is defined as:

$$
C_{v o}(z)=\frac{\beta}{\left(1+\beta m_{11}\right)\left(1+\beta m_{22}\right)-\left(\beta^{2} m_{12} m_{21}\right)}\left(\begin{array}{cc}
1+\beta m_{22} & -\beta m_{12} \\
-\beta m_{21} & 1+\beta m_{11}
\end{array}\right)
$$

With a sufficiently high chosen of $b$, we obtain:

$$
\begin{aligned}
C_{v o}(z) & \simeq \frac{1}{\left(m_{11} m_{22}-m_{12} m_{21}\right)}\left(\begin{array}{cc}
m_{22} & -m_{12} \\
-m_{21} & m_{11}
\end{array}\right) \\
& \simeq \frac{\operatorname{com}(M(z))}{\operatorname{det}(M(z))} \\
& \simeq M(z)^{-1}
\end{aligned}
$$

This result coincides with that obtained by equation (6).

The block diagram of the IMC structure, proposed in the Figure 4 where the inversion approach is that described in this section. case of discrete over-actuated systems, is illustrated in

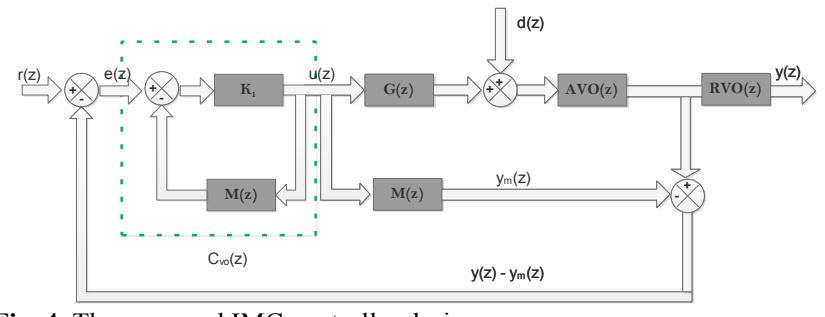

Fig. 4. The proposed IMC controller design

According to the Figure 4, we can deduce the equations (9) and (10) that describe respectively the output vector $y(z)$ ( $\left.y(z)=y_{r}(z) r(z)+y_{d}(z) d(z)\right)$ without considering the blocks $A V O(z)$ and $R V O(z)$ :

$y_{r}(z)=G(z)\left(\mathrm{I}_{m}+\left(\mathrm{I}_{m}+K_{1} M(z)\right)^{-1} K_{1}(G(z)-M(z))\right)^{-1}\left(\mathrm{I}_{m}+K_{1} M(z)\right)^{-1} K_{1}$ 


$$
y_{d}(z)=I_{m}-G(z)\left(\mathrm{I}_{m}+\left(\mathrm{I}_{m}+K_{1} M(z)\right)^{-1} K_{1}(G(z)-M(z))\right)^{-1}\left(\mathrm{I}_{m}+K_{1} M(z)\right)^{-1} K_{1}
$$

When the model is perfect and if we assume that the process is not subject to any disturbance, the output vector becomes:

$$
\begin{aligned}
y(z) & =G(z)\left(I_{m}+K_{1} G(z)\right)^{-1} K_{1} r(z) \\
& =M(z)\left(I_{m}+K_{1} M(z)\right)^{-1} K_{1} r(z)
\end{aligned}
$$

This expression (11) allows us to conclude that the robustness of the proposed internal controller approach depends only of the matrix gain $K_{1}$ and $M(z)$.

For the case of systems with NMP and/or delay, the coefficient $b$ cannot be chosen sufficiently high, this can lead to degradation of system accuracy. In fact, in order to ensure the system accuracy, i.e., a zero static error, the controller static gain matrix $C_{v o}(1)$ must be very close to the inverse of the model static gain matrix $M(1)$ as shown in (12).

$$
C_{V O}(1)=M(1)^{-1}
$$

In order to remedy this problem, the gain matrix $K_{2}$ presented in Figure 4 is added. It allows to compensate the system static errors. Its expression is given by (13) and it ensures that $C_{V O}(z) M(z)=I_{m}$.

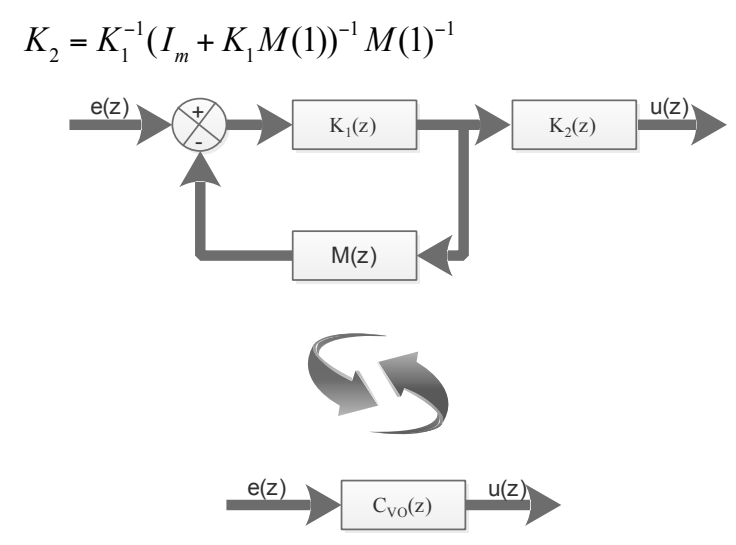

Figure 5. Generalized Virtual Output Controller structure

$K_{2}$ is used to compensate the static errors, while $K_{1}$ is chosen to ensure the stability of the loop to achieve the goal of the inversion.

The new generalized Virtual Outputs Controller expression for over-actuated systems with NMP is the following:

$$
C_{v o}(z)=K_{2}\left(I_{m}+K_{1} M(z)\right)^{-1} K_{1}
$$

\subsection{Study of the stability of the controller $C_{v o}(z)$}

The IMC controller $C_{v o}(z)$ expressed by equation (14) can be reforumlated using the state space representation as follows:

$$
\left\{\begin{array}{l}
x_{c}(k+1)=F_{c} x(k)+H_{c} e(t) \\
\mathrm{y}_{c}(K)=C_{c} x(K)
\end{array}\right.
$$

where: $x(k) \in R^{n}$ and $y(k) \in R^{m}$ are respectively the state vector and the system output vector. The matrices $F_{c}, H_{c}$ and $C_{c}$ are known constant matrices. The stability analysis can be processed by the knowledge of the state matrix $F_{c}$ which depends of the gain matrix $K_{l}$.

The system presented by equation (15), is asymptotically stable in the Lyapunov sense [22], if there exists a positive definite matrix $P=P^{T}>0$, satisfying the following Lyapunov inequality.

$$
P>0, P F_{c}+P^{T} F_{c}<0
$$

\section{Uncertain system stability}

Many sources may influence processes during their lifecycles, such as the variation of real parameters or the inherent non-linearity characteristic. In this paper, we give a possible approach to solve the problem of robust stability of a family of interval polynomials, whose parameters are stochastically and independently varying in some given range, using a simple graphical approach [23].

The parametric description of uncertainty is given by a known structure but imprecise knowledge of real physical parameters of the system. Their possible values are usually bounded by intervals. We first give the form of interval polynomials:

$$
P(\mathrm{z}, \mathrm{q})=\sum_{i=0}^{n} p\left(\mathrm{q}_{n-i}\right) \mathrm{z}^{i}, q \in Q=\left[\underline{q}_{n-i} \leq q_{n-i} \leq \bar{q}_{n-i}\right]
$$

where $z$ is the complex variable, $q$ is the vector of uncertain parameters, and $n$ is the order of the system. The discrete-time characteristic polynomials (17) is stable if and only if $P(z, q)$ is stable for all $q \in Q$, i.e. all roots of $P(z, q)$ are located inside the unit circle. The entries $q_{n-i}$ of $P(\mathrm{z}, \mathrm{q})$ are independent and have uncertainty bounds $\underline{q}_{n-i}$ and $\bar{q}_{n-i}$ respectively.

The direct calculation of the roots can be impractical, so more efficient technique to analyze the stability are used. Among existing methods [18], [23], the graphical approach based on the combination of the value set concept and the zero-exclusion condition is used in this paper.

The value set construction requires to substitute $z$ in $P(z, q)$ with $j w$, then we fix the frequency $w$ and let the vector $q$ range over the set $Q$. The zero exclusion condition states that, the uncertain characteristic polynomial $P$ is robustly stable if and only if the complex plane origin is excluded from the value set $P(j w, Q)$, at all frequencies $j w$, i.e. $P$ is robustly stable if and only if $0 \notin P(j \omega, Q) \forall \omega \geq 0$.

The IMC structure, whose fundamentals are described 
Islem Bejaoui, Imen Saidi, Maria Gabriella Xibilia and Dhaou Soudani/

Journal of Engineering Science and Technology Review 12 (2) (2019) 111 - 118

here, have to offer as good a performance as can be achieved by an "optimal internal controller". The quality of the designed controller should be judged according to the following criteria:

- Regulatory behavior: the output variables are to be kept at their set point despite disturbance affecting the system;

- Robustness: stability and acceptable control performance should be maintained in the face of all the changes in the process model;

- Servo behavior: changes in the set point should be tracked fast;

- Ability to deal with constraints on the inputs,

$G(p)=\left(\begin{array}{lll}g_{11} & g_{12} & g_{13} \\ g_{21} & g_{22} & g_{23}\end{array}\right)=\left(\begin{array}{lll}\frac{1}{s+1} e^{-s} & \frac{1}{(s+1)(s+3)} e^{-2 s} & \frac{1}{s+3} e^{-2 s} \\ \frac{1}{s+1} e^{-s} & \frac{s-2}{(s+1)(s+3)} e^{-2 s} & \frac{1}{s+3} e^{-2 s}\end{array}\right)$

Let us consider the case of perfect modeling $G(p)=M(p)$. The augmented model transfer matrix is of dimensions $(m \times m)$, where the model transfer functions $m_{11}, m_{12}, m_{13}, m_{21}, m_{22}$ and $m_{23}$ are chosen equal to $g_{11}$, $g_{12}, g_{13}, g_{21}, g_{22}$ and $g_{23}$. The augmenting $((m-n), m)$ states and outputs.

The effectiveness of the approach is shown in the following section trough a numerical example.

\section{Numerical Example}

In this section, an example is considered to demonstrate the possibility of the proposed approach. A non-square system with time delays and NMP is considered which has 3 inputs and 2 outputs, where the transfer function matrix is given as [24]:

$$
M(p)=\left(\begin{array}{ccc}
m_{11} & m_{12} & m_{13} \\
m_{21} & m_{22} & m_{23} \\
m_{31} & m_{32} & m_{33}
\end{array}\right)=\left(\begin{array}{ccc}
\frac{1}{s+1} e^{-s} & \frac{1}{(s+1)(s+3)} e^{-2 s} & \frac{1}{s+3} e^{-2 s} \\
\frac{1}{s+1} e^{-s} & \frac{s-2}{(s+1)(s+3)} e^{-2 s} & \frac{1}{s+3} e^{-2 s} \\
\alpha & \alpha & \alpha
\end{array}\right)
$$

The bilinear method of discretization is applied for the process $G(p)$ and the model $M(p)$. The interval of the gain $K_{1}$ which assures the stability of the IMC structure is $0 \leq K_{1} \leq 1.5 \times I_{3}$ which satisfies the necessary conditions of stability previously mentioned with LMI approach. Two different value are considered, corresponding to $b=0.1$ and $b=0.9$. We can notice that the value of $b$ is not high enough because we consider an NMP system with timedelay. by:

The gain matrix $K_{2}$ relative to $K_{1}=0.1 \times I_{3}$ is given

$$
K_{2}=\left(\begin{array}{ccc}
0.798 & -0.199 & -1.9948 \\
0.1 & -0.1 & 0 \\
-2.1942 & 0.6986 & 5.985
\end{array}\right)
$$

The gain matrix $K_{2}$ relative to $K_{1}=0.9 \times I_{3}$ is given by: virtual transfer functions $m_{31}, m_{32}$ and $m_{33}$, can be simply chosen as constant $\mathrm{a}$.

The transfer function of the augmented process model using the virtual outputs approach is given as:

$$
K_{2}=\left(\begin{array}{ccc}
9.866 & -2.2167 & -22.1646 \\
1.1112 & -0.1112 & 0 \\
-24.38 & 7.7619 & 67.5004
\end{array}\right)
$$

Figure 7 show the system outputs signal $y_{1}$ and $y_{2}$ in the case where we use the virtual outputs controller $C_{v o}(z)$ for two different values of $\beta$ as previously mentioned.

It can be shown in these, that the system control effect is not satisfactory, for the output $y_{1}$ the static error is $\varepsilon_{1}(\infty)=0.17$ for $b=0.1$ and $\varepsilon_{1}(\infty)=0.8$ for $b=0.9$

the output $y_{2}$ the static error is $\varepsilon_{1}(\infty)=0.05$ for $b=0.1$ and $\varepsilon_{2}(\infty)=0.37$ for $b=0.1$, so for the both values of $b$ the system has not good tracking. These two simulations show the effect of the choice of $b$, the more this last increase, the more the system gains in precision and speed. 
Islem Bejaoui, Imen Saidi, Maria Gabriella Xibilia and Dhaou Soudani/

Journal of Engineering Science and Technology Review 12 (2) (2019) 111 - 118

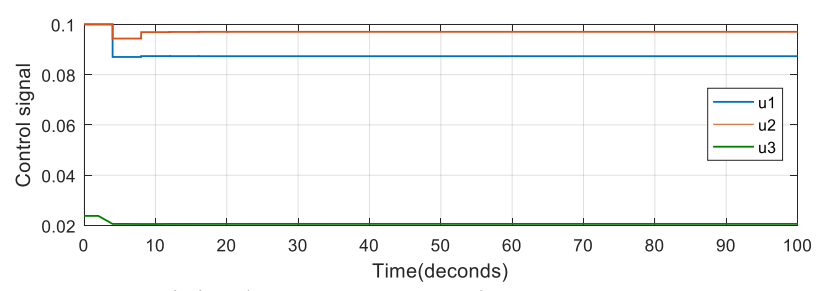

Fig. 6. Control signal, NMP over-actuated system

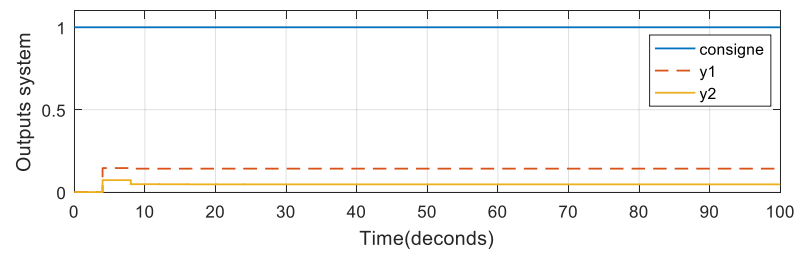

(a)

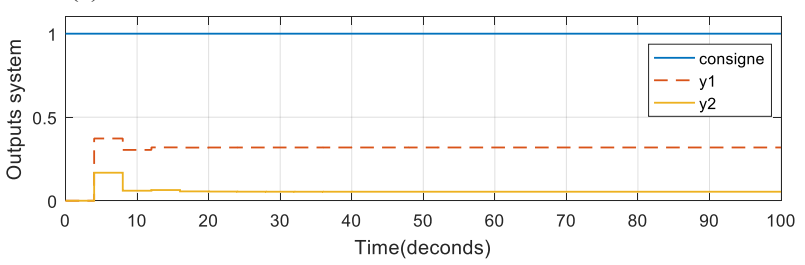

(b)

Fig. 7. System outputs, NMP over-actuated system: (a) Step response of the system for $b=0.1(b)$ Step response of the system for $b=0.9$.

Figure. 9 show the system outputs signals using the gain matrix $K_{2}$ in the cases $\mathrm{b}=0.1$ and $\mathrm{b}=0.9$, respectively. We can note that the system responses $\mathrm{y}_{1}$ and $\mathrm{y}_{2}$ start negatively with a little delay because the system is non-minimum phase with time-delays and we can notify than, the more the value of $b$ increases, the more the peak in the transitional regime is high. We can notice that the insertion of the gain matrix $K_{2}$ has notably improved the precision.

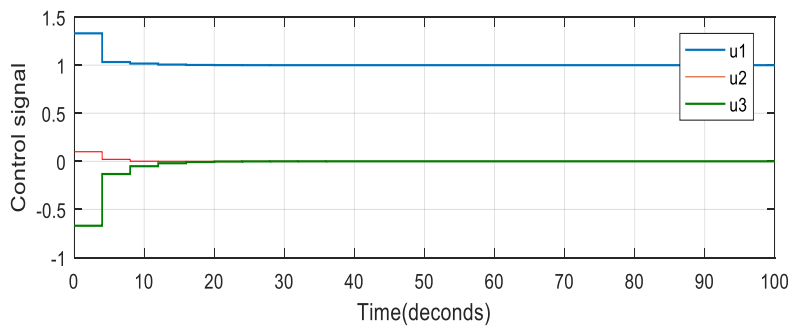

Fig. 8. Control signal, NMP over-actuated system, presence of $\mathrm{K}_{2}$

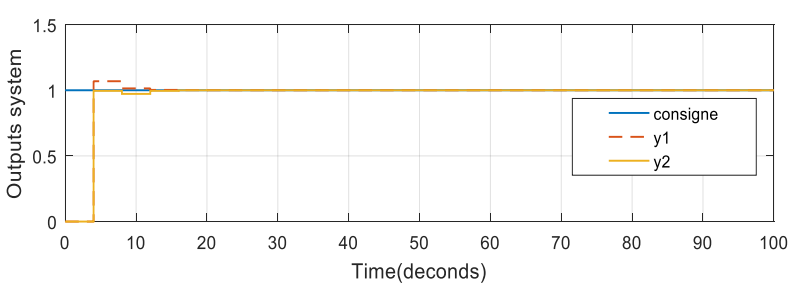

(a)

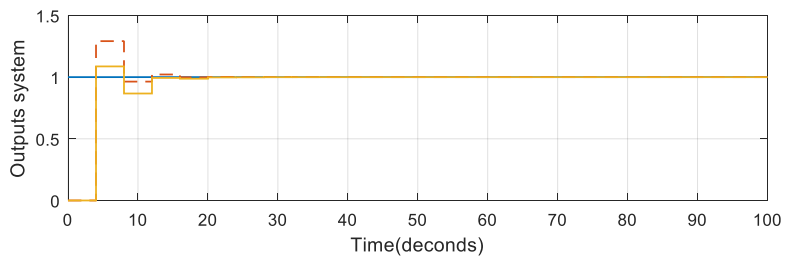

(b)

Fig. 9. System outputs, NMP over-actuated system, presence of $K_{2}$ (a) Step response of the system for $b=0.1$ (b) Step response of the system for $b=0.9$.

The response of the perfect controller $C_{V O}(z)=M(\mathrm{z})^{-1}$ to the set-point is shown in Figure 9. This deadbeat response shown is obtained through relatively strong input action. A tuning parameter $b$ which has a direct effect on the closed-loop response can alleviate the strong input action (Figure 6,8).

Results prove the effectiveness of the presented approach to ensure a fast set-point tracking and to preserve the system performances in spite of the presence of disturbance and non-minimum phase with time-delays behavior. In the following the effect of disturbance and parameters variations are considered.

In order to validate the robustness of the proposed IMC structure disturbance-rejection, step disturbance signals $d_{1}$, $d_{2}$ and $d_{3}$ are applied to the system at different sampling times 20,40 and 60 .

$$
d(z)=\left[\begin{array}{lll}
d_{1} & d_{2} & d_{3}
\end{array}\right]^{T}, d_{1}=d_{2}=d_{3}=\frac{0.2}{z-1}
$$

According to the Figure 10 (below), the output response $y_{1}$ and $y_{2}$ track quickly the set values. Even in the presence of external disturbance, the IMC structure still maintained a good behavior.

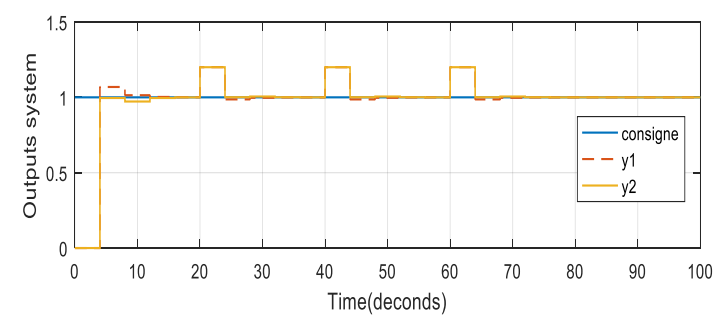

Fig. 10. System outputs, NMP over-actuated system, presence of disturbance

Let us consider a possible uncertainty in the process parameters, more precisely at the level of the characteristic polynomial. Consider the same example (18) of discrete multivariable system subjected to parameters variation, defined by:

$$
G(z)=\left(\begin{array}{lll}
g_{11}(z) & g_{12}(z) & g_{13}(z) \\
g_{21}(z) & g_{22}(z) & g_{23}(z)
\end{array}\right)=\left[\begin{array}{ccc}
\frac{N_{11}}{P_{11}} & \frac{N_{12}}{P_{12}} & \frac{N_{13}}{D_{13}} \\
\frac{N_{21}}{D_{21}} & \frac{N_{22}}{P_{22}} & \frac{N_{23}}{P_{23}}
\end{array}\right]
$$

where, the discrete-time characteristic polynomials $P_{n m}$ with uncertainty of the process are the following:

$$
\begin{aligned}
& P_{11}(z)=[0.4,1.2] z-0.01832 \\
& P_{12}(z)=[0.5,1.5] z^{2}-[0.1,2.9] z+0.00004076 \\
& P_{22}(z)=[0.9,3] z^{2}-[0.01,0.19] z+0.04 \\
& P_{23}(z)=[0.7,1.8] z-0.09072
\end{aligned}
$$


Islem Bejaoui, Imen Saidi, Maria Gabriella Xibilia and Dhaou Soudani/

Journal of Engineering Science and Technology Review 12 (2) (2019) 111 - 118

In the following, parametric open loop stability is considered and each characteristic polynomials for the transfer function matrix of $g_{11}(z), g_{12}(z), g_{22}(z)$ and $g_{23}(z)$, are replaced by $P_{11}(z), P_{12}(z), P_{22}(z)$ and $P_{23}(z)$, respectively. The value sets are plotted by considering frequency $\omega \in\left[\begin{array}{ll}0 & 3\end{array}\right]$ with step 0.2 .

From Figure 11, the value sets of each family $g_{11}(z)$, $g_{12}(z), g_{22}(z)$ and $g_{23}(z)$, does not included the origin $(0,0)$ of the unit circle for all frequencies. The zero-exclusion condition for discrete time interval polynomials is verified.

The influence of such uncertainties is shown by Figure 12; we can conclude that the tracking set and the accuracy of the system are maintained despite the uncertainties. Then one can say that the proposed IMC structure ensures the satisfaction of a certain level of performance despite the presence of uncertainties.

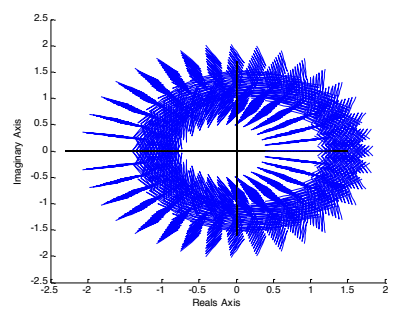

(a) Stability region for $g_{11}(z)$

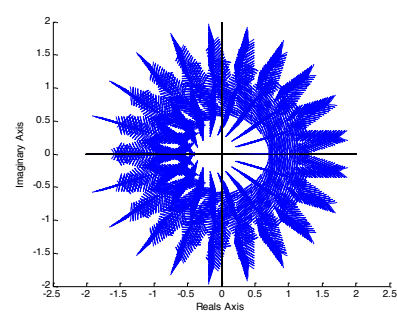

(c) Stability region for $g_{22}(z)$

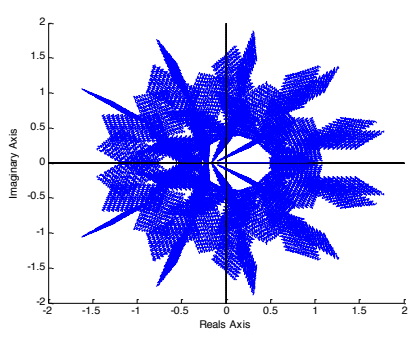

(b) Stability region for $g_{12}(z)$

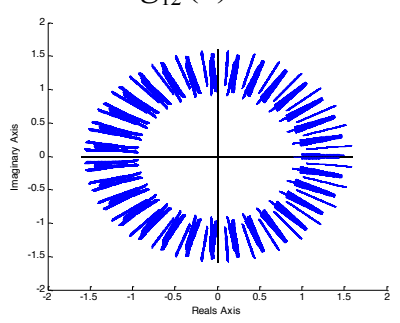

(d) Stability region for $g_{23}(z)$

Fig. 11. The zero-exclusion condition of a family of interval polynomials "The zero-exclusion condition" approach - robustly stable case
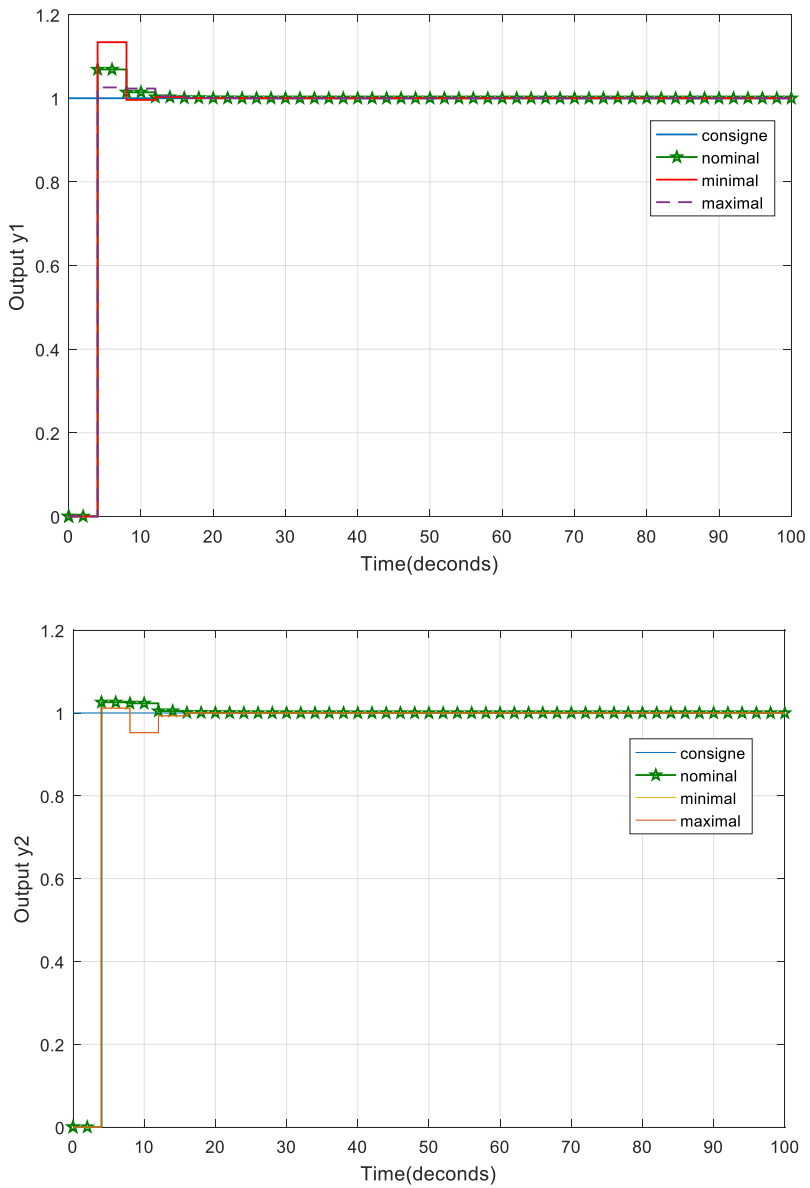

Fig. 12. Control responses of an NMP over-actuated system with interval parametric uncertainty

\section{Conclusion}

The paper presents a new IMC methodology for uncertain over-actuated multivariable systems with time delays and non-minimum phase zeros. The need of a new method is required because the inversion of the transfer matrix cannot be computed for over-actuated system. Two blocks, denoted as $A V O$ and $R V O$ have been inserted in the basic IMC scheme. The first block is used to augment the original system and make it invertible. The augmented outputs are then removed by the $R V O$ block. The method proposed in the paper allowed to obtain good performance even in presence of parameters uncertainties. According to all the simulations, we notice that the outputs of the system reach quickly and without exceeding their established regimes. These satisfactory results show the capacity of the new IMC methodology to maintain its performance despite the uncertainty parameters and time delays. The same methodology can be used in the case of underactuated systems.

This is an Open Access article distributed under the terms of the Creative Commons Attribution License

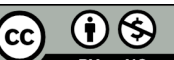

References 
Islem Bejaoui, Imen Saidi, Maria Gabriella Xibilia and Dhaou Soudani/

Journal of Engineering Science and Technology Review 12 (2) (2019) 111 - 118

1. N.P.I. Aneke, "Control of underactuated mechanical systems," Eindhoven: Technische Universiteit, Published: 01/01/2003.

2. C. I. Muresan, E. H. Dulf, C. Copot, R. De. Keyser and C. Ionescu, "Design and analysis of a multivariable fractional order controller for a non-minimum phase system," Journal of Vibration and Control, pp. 1-9, 2015.

3. S. Bose, Y. V. Hote and S. D. Hanwate, "Analysis of practical non-minimum phase systems by transfer function approach," 4th International Conference on Electrical Energy Systems (ICEES), Chennai, pp. 500$505,2018$.

4. L. Qiu and E. J. Davison, "Performance limitations of non-minimum phase systems in the servomechanism problem," Automatica, vol. 29, no. 2, pp. 337-349, 1993.

5. C. E. Garcia and M. Morari, "Internal model control. A unifying review and some new results," Industrial \& Engineering Chemistry Process Design and Development, 21 (2), 308-323, 1982.

6. S. Datta, U. M. Nath and C. Dey, "Design and implementation of decentralized IMC-PI controllers for real time coupled tank process," Michael Faraday IET International Summit 2015, pp. 93-98 Kolkata, 2015.

7. X. Sun, Z. Shi, L. Chen and Z. Yang, "Internal Model Control for a Bearingless Permanent Magnet Synchronous Motor Based on Inverse System Method," IEEE Transactions on Energy Conversion, vol. 31, no. 4, pp. 1539-1548, Dec. 2016.

8. W. Tan and C. Fu, "Linear Active Disturbance-Rejection Control: Analysis and Tuning via IMC," IEEE Transactions on Industrial Electronics, vol. 63, no. 4, pp. 2350-2359, April 2016.

9. T. Kobaku, S. C. Patwardhan and V. Agarwal, "Experimental Evaluation of Internal Model Control Scheme on a DC-DC Boost Converter Exhibiting Nonminimum Phase Behavior," IEEE Transactions on Power Electronics, vol. 32, no. 11, pp. 8880-8891, Nov. 2017.

10. P. P. Arya and S. Chakrabarty, "IMC based Fractional Order Controller Design for Specific Non-Minimum Phase Systems," IFAC-PapersOnLine, vol.51, no 4, pp. 847-852, 2018.

11. P. Garcia, P. Albertos and T. Hagglund, "Control of unstable non-minimum-phase delayed systems," Journal of Process Control 16 (2006) 1099-1111, 2006.

12. J. Qibing, G. Yan, L. Ziyi and S. Anan, "Decoupling Internal Model Control for Non-square Process with Time Delays ,'International Conference on Measuring Technology and Mechatronics Automation.2010.
13. J-C Liu, N. Chen, X. Yu., "Modified Two-Degrees-ofFreedom Internal Model Control for Non-Square Systems with Multiple Time Delays," Journal of Harbin Institute of Technology (new series), Vols.21, No.2 China, pp. 122-128, 2014.

14. Ricardo Cajo, Shiquan Zhao, Clara M. Ionescu, Robin De Keyser, Douglas Plaza, Sheng Liu, "IMC based PID Control Applied to the Benchmark PID18, "IFACPapersOnLine, vol. 51, no. 4, pp. 728-732, 2018.

15. I. Bejaoui, I. Saidi, D. Soudani, "New internal model controller design for discrete over-actuated multivariable system," IEEE 4th International Conference on Control Engineering and Information Technology (CEIT),pp.1-6, Hamamet, Tunisia,2016.

16. A. Fossard, "Commande des systémes multidimensionnels," Technique de l'automatisme, Dunod, Paris 1972.

17. S. Boyd, L.El. Ghaoui, E. Feron, V. Balakrishnan, "Linear Matrix Inequalities in System and Control Theory," Philadel-phia: SIAM Press, 1994.

18. R. Matusu, "Robust Stability Analysis of Discrete-Time Sytems with Parametric Uncertainty: A Graphical Approach," International Journal of Mathematical Models and Methods in Applied Sciences, vol.8, pp. 95$102,2014$.

19. I. Bejaoui, I. Saidi, and D. Soudani, "New Internal Model Controller design for discrete over-actuated multivariable system," 4th International Conference on Control Engineering \& Information Technology (CEIT), Hamamet, 2016.

20. I. Bejaoui, I. Saidi, and D. Soudani, “ Internal model stability analysis,", International Journal of Control Theory and Application (IJCTA).10(34): 139-150, 2017.

21. Dhahri, A., Saidi, I., and Soudani, D, "A new internal model control method for MIMO over-actuated systems," International Journal of Advanced Computer Science and Applications, 7 (10), 2016.

22. S. Dussy, "Robust Diagonal Stabilization: An LMI Approach," IEEE Transactions On Automatic Control, Vol. 45, 2000.

23. R. Matusu, Roman Prokop, "Robust Stability Analysis for Systems with Real Parametric Uncertainty: Implementation of Graphical Tests in Matlab," International Journal of Circuits Systems and Signals, Processing. Issue 1, Volume 7, 2013.

24. J.C. Liu, N. Chen and X. Yu, "Modified two-Degrees-offreedom internal model control for non-square systems with multiple time delays," Journal of Harbin Institue of Technology (New Series), vol.21 No.2, 2014. 